\title{
Vibrations of cables with bending stiffness by an asymptotic approach
}

\author{
T. Canor \\ F.R.S.-FNRS, National Fund for Scientific Research of Belgium \\ ArGEnCo Department, University of Liege, Belgium
}

V. Denoël

ArGEnCo Department, Structural Engineering Division

University of Liege, Belgium

\begin{abstract}
Vibrations of cables with a small bending stiffness concerns many engineering applications such as the fatigue assessment of stay cables. With the finite element (FE) method, the analysis can be performed with nonlinear truss elements, but bending effects are not taken into account. Otherwise, beam elements can be used, but the smallness of the bending stiffness may lead to numerical instability and mediocre results in boundary regions.

In this context, the paper presents an alternative method to calculate the time evolution of the profile of bending moments in boundary layers of cables, avoiding heavy FE analysis. The developments combine the theory of vibrations of extensible rods with asymptotic methods. The equations are decoupled between the slow dynamics of the boundary regions and the fast dynamics of the span. Then, a composite solution is constructed by means of a matched asymptotic procedure.
\end{abstract}

\section{INTRODUCTION}

The vibration analysis of cables with a small bending stiffness is a problem encountered in many engineering applications such as the fatigue assessment of stay cables (cable stayed bridges or prestressed concrete beams), the modeling of pipeline laying operation or the determination of bending stresses in drillpipe assemblies. The elastic theory of rods models the static and dynamic deflections of general elastic beams and cables (Antman 2005, Coleman et al. 1993). However, the smallness of the bending stiffness leads to consider a singularly perturbed equations and the existence of boundary layers near the anchors (Hinch 1991). In these small regions, the curvature and the bending moment change sharply compared with the rest of the cable especially. This phenomenon is magnified for clamped beams. Standard numerical techniques fail to solve these problems efficiently.

Static deflection of cables with a small bending stiffness is a widely covered topic. A matched asymptotic expansion has been used (Stump \& van der Heijden 2000) to study the spatial deflection of a bent and twisted inextensible cable. Denoël \& Detournay (2010) propose an application of multiple scales methods to study a beam with a small bending stiffness, offering a better accuracy for a larger range of bending stiffness than the matched asymptotic method. From another viewpoint, Denoël \& Canor (2013) have developed a patching method allowing to quantify the size of the boundary layer of the cable using an Eulerian description of a catenary in the span and a nonlinear beam model in the boundary layers.

Concerning nonlinear vibration of cables, the finite element method widely used in structural dynamics presents some weaknesses. The use of nonlinear truss elements (Impollonia et al. 2011) neglects completely the bending effects, while nonlinear beam elements may lead to instability because of the smallness of the bending stiffness. Mediocre results can be obtained for indirect excitations in boundary layers. These zones in which the bending moment increases sharply, require an expensive mesh refinement with small elements which leads to increase the computational burden or to have ill-conditioned systems. In the range of analytical or semi-analytical methods, Stump \& Fraser (2000) have used matched asymptotic expansion to study the boundary layer behaviour of moving strips in a steady state deflection (no inertial forces). Pellicano et al. (1998) study the dynamical behaviour of an axially moving beam with hinged extremities: a linear modal analysis associated with matched asymptotic expansion allows to decouple quite efficiently the equation of the beam, eventhough 
the potential nonlinear bending behaviour is not considered. The modal analysis for cable with small bending stiffness is also presented in the works of Ricciardi et al. (2008) and Lacarbonara et al. (2008). The modal approach may seem attractive, nevertheless the complex analytical shape of modes is a drawback just to take into account small perturbations in the boundary regions. Furthermore, modal analysis of slack cables is usually based on rough approximations.

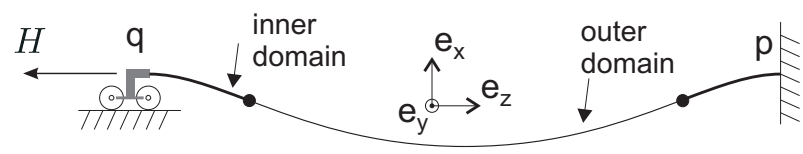

Figure 1: Sketch of problem

As an alternative to numerical methods and modal analysis, provided the complexity of the analytical developments does not preclude their application, these problems may be tackled with appealing analytical procedures such as matched asymptotic or multiple scales methods (Hinch 1991). This work proposes to start with classical theory of rod vibration and to use the potentiality of asymptotic methods to derive an efficient and smart formulation to analyse the vibration of taut and clamped cable with bending stiffness as sketched in Figure 1.

\section{FORMULATION OF THE PROBLEM}

\subsection{Theory of extensible rods}

The classical theory of rods (Antman 2005) models the motion of elastic structures submitted to external forces in large displacements and large rotations. The rod deflection is described with a configuration vector $\mathbf{r}(s, t)$ which defines a curve $\mathcal{C}(t)$. The variable $s$ identifies the position of a material point of the rod in the stress-free reference configuration $\mathbf{r}^{0}(s)$, while $t$ denotes the time. The function $\mathbf{r}(s, t):[0, l] \times \mathbb{R}^{+} \rightarrow \mathbb{R}^{3}$ is a continuous function with $l$ the length of the rod in $\mathbf{r}^{0}(s)$. The tangent vector to $\mathcal{C}(t)$, noted $\partial_{s} \mathbf{r}$, is defined as the strain vector $\mathbf{v}$ such that $\partial_{s} \mathbf{r}=\mathbf{v}$. The norm of $\mathbf{v}$ is usually called the stretch.

A local basis, different from the Frenet basis related to the curve $\mathcal{C}(t)$ at a point $s$, is usually introduced. The cross section is determined by the orientation of two orthogonal vectors $\tilde{\mathbf{d}}_{1}(s, t)$ and $\tilde{\mathbf{d}}_{2}(s, t)$. The local basis is completed by the definition of a third vector $\tilde{\mathbf{d}}_{3}(s, t)=\tilde{\mathbf{d}}_{1} \times \tilde{\mathbf{d}}_{2}$ which is normal to the surface of the cross section of the rod. If the rod in unshearable but extensible, the strain vector in the local basis has a single component defined such that $\mathbf{v}=\left[0,0,1+v_{3}\right]^{T}$ with $\left.\left.v_{3} \in\right]-1,+\infty\right]$ the strain in the axial direction of the rod.

The dynamics of rods is modelled by a set of partial differential equations in space and time. The conservation of linear and angular momenta yields

$\partial_{s} \mathbf{N}+\mathbf{F}=\rho A \partial_{t}^{2} \mathbf{r}$ $\partial_{s} \mathbf{M}+\partial_{s} \mathbf{r} \times \mathbf{N}=\rho I\left(\tilde{\mathbf{d}}_{1} \times \partial_{t}^{2} \tilde{\mathbf{d}}_{1}+\tilde{\mathbf{d}}_{2} \times \partial_{t}^{2} \tilde{\mathbf{d}}_{2}\right)$

where $\mathbf{M}(s, t)$ and $\mathbf{N}(s, t)$ are the vectors of internal moments and forces for a material point $s$ at a time $t$, respectively. Furthermore, $\rho, A$ and $I$ are the mass density of the beam material, the cross section area and the second moment of area per unit of reference length. The parameters of the cable are independent of $t$ and $s$. The section is supposed to be circular, so the second moment of area is independent of $\tilde{\mathbf{d}}_{1}$ and $\tilde{\mathbf{d}}_{2}$ as principal axes.

The conservation of planar cross section, or the Euler-Bernoulli hypothesis (Bernoulli 1694), is commonly assumed in rods and cables when the shear forces do not induce significant strain energy. The constitutive equation for internal moments with this assumption yields

$\mathbf{M}=E I\left(\kappa_{1} \tilde{\mathbf{d}}_{1}+\kappa_{2} \tilde{\mathbf{d}}_{2}\right)+G J \kappa_{3} \tilde{\mathbf{d}}_{3}$

with $E$ the Young modulus, $G$ the shear modulus, $G J$ torsional stiffness, $\kappa_{1,2}$ the curvature in the direction $\tilde{\mathbf{d}}_{1,2}$ and $\kappa_{3}$ the twist density. The constitutive equation for internal forces takes into account the extensibility and the unshearability of the rods, such that

$$
N_{3}=E A v_{3} .
$$

At this stage, because of the assumption of unshearability, the complete set of 9 equations involves 9 unknowns, which leads to a well-posed problem.

\subsection{Euler angles}

Euler angles (Antman 2005, Coleman et al. 1993) can be interpreted as a generalization of the spherical coordinates. In the theory of elastic rods, they are often used because they provide an efficient description of the orientation of a cross section compared with a reference system, in spite of some drawbacks, as discontinuities. The method consists in mapping the fixed basis $\left\{\mathbf{e}_{x}, \mathbf{e}_{y}, \mathbf{e}_{z}\right\}$ with the local basis of the cross section by a set of three successive rotations of angles $\psi(s, t)$ (precession), $\theta(s, t)$ (nutation) and $\phi(s, t)$ (spin). In the reference configuration $\mathbf{r}^{0}(s)$, the axis of the rod lies on the $\mathbf{e}_{z}$-axis and the two principal axes of the cross section are parallel to $\mathbf{e}_{x^{-}}$and $\mathbf{e}_{y^{-}}$-axes.

The first two rotations match the vector $\mathbf{e}_{z}$ with the unit vector $\tilde{\mathbf{d}}_{3}(s, t)$ normal to the cross section of the rod. In spherical coordinates, this normal vector reads

$\tilde{\mathbf{d}}_{3}=\cos \psi \sin \theta \mathbf{e}_{x}+\sin \psi \sin \theta \mathbf{e}_{y}+\cos \theta \mathbf{e}_{z}$.

The two unit vectors $\tilde{\mathbf{d}}_{1}(s, t)$ and $\tilde{\mathbf{d}}_{2}(s, t)$ at abscissa $s$ are contained in a plane perpendicular to $\tilde{\mathbf{d}}_{3}(s, t)$. The transformation between the fixed basis and the local basis is completed by a last rotation $\phi(s, t)$ around $\tilde{\mathbf{d}}_{3}(s, t)$ such that $\tilde{\mathbf{d}}_{1}(s, t)$ and $\tilde{\mathbf{d}}_{2}(s, t)$ are expressed in terms of $\psi(s, t), \theta(s, t)$ and $\phi(s, t)$ (see (Antman 
2005)). At this stage, the nine components of the local basis depend on three angles only. The curvature vector in terms of Euler angles reads

$$
\begin{aligned}
\boldsymbol{\kappa}= & \left(\sin \phi \partial_{s} \theta-\cos \phi \sin \theta \partial_{s} \psi\right) \tilde{\mathbf{d}}_{1}+\left(\cos \phi \partial_{s} \theta\right. \\
& \left.+\sin \phi \sin \theta \partial_{s} \psi\right) \tilde{\mathbf{d}}_{2}+\left(\cos \theta \partial_{s} \psi+\partial_{s} \phi\right) \tilde{\mathbf{d}}_{3} .
\end{aligned}
$$

However, because the cable is reasonably assumed to have a circular cross section, the orientation of $\tilde{\mathbf{d}}_{1}(s, t)$ and $\tilde{\mathbf{d}}_{2}(s, t)$ is independent of the rotation $\phi(s, t)$, because every couple of orthogonal unit vectors normal to $\tilde{\mathbf{d}}_{3}(s, t)$ are principal axes (Coleman et al. 1993). A new local basis may be defined by imposing a clockwise rotation $\phi(s, t)$ around $\tilde{\mathbf{d}}_{3}(s, t)$ to the couple $\tilde{\mathbf{d}}_{1}(s, t)$ and $\tilde{\mathbf{d}}_{2}(s, t)$, such that

$$
\begin{aligned}
& \mathbf{d}_{1}=\cos \psi \cos \theta \mathbf{e}_{x}+\sin \psi \cos \theta \mathbf{e}_{y}-\sin \theta \mathbf{e}_{z} \\
& \mathbf{d}_{2}=-\sin \psi \mathbf{e}_{x}+\cos \psi \mathbf{e}_{y}
\end{aligned}
$$

and $\mathbf{d}_{3}=\tilde{\mathbf{d}}_{3}$. The same clockwise rotation imposed to the curvature vector yields

$\boldsymbol{\kappa}=\left(\cos \theta \partial_{s} \psi+\partial_{s} \phi\right) \mathbf{d}_{3}+\partial_{s} \theta \mathbf{d}_{2}-\sin \theta \partial_{s} \psi \mathbf{d}_{1}$.

\subsection{Dimensionless formulation}

A dimensionless formulation is introduced. Dimensionless vectors, variables and parameters are mentioned with Greek letters and bar symbols. The material coordinate $s$ is scaled with respect to the stressfree rod length $l$, such as $\xi=s / l$ with $\xi \in[0,1]$. The internal and external forces $\mathbf{N}$ and $\mathbf{F}$ are scaled by the total weight of the rod $w l$ and the bending moment $\mathbf{M}$ is scaled by $w l^{2} ; w$ is the weight per unit of length. This choice is motivated by the time-invariance of $w$ and $l$. The dimensionless time $\tau$ is obtained by scaling the time $t$ with a reference period $T_{1}=\sqrt{l / g}$ with $g$ the gravitational acceleration. The dimensionless bending stiffness is given by $\varepsilon^{2}=E I / w l^{3}$ and the position vector $\mathbf{r}$ is scaled by $l$. A parameter $\varepsilon_{b}=w l / E A$ is introduced in the constitutive equation of extensibility and a parameter $\gamma$ is defined as the ratio between the torsional stiffness $G J$ and the bending stiffness EI.

The kinematic condition of unshearability but extensibility implies $\partial_{\xi} \overline{\mathbf{r}}=\left(1+v_{3}\right) \mathbf{d}_{3}$ and the curvature vector becomes

$\overline{\boldsymbol{\kappa}}=\left(\cos \theta \partial_{\xi} \psi+\partial_{\xi} \phi\right) \mathbf{d}_{3}+\partial_{\xi} \theta \mathbf{d}_{2}-\sin \theta \partial_{\xi} \psi \mathbf{d}_{1}$

with $\overline{\boldsymbol{\kappa}}=l \boldsymbol{\kappa}$. Then, the equations of motion become

$\partial_{\xi} \overline{\mathbf{N}}+\overline{\mathbf{F}}=\partial_{\tau}^{2} \overline{\mathbf{r}}$

$\partial_{\xi} \overline{\mathbf{M}}+\partial_{\xi} \overline{\mathbf{r}} \times \overline{\mathbf{N}}=\varepsilon^{2} \varepsilon_{b}\left(\mathbf{d}_{1} \times \partial_{\tau}^{2} \mathbf{d}_{1}+\mathbf{d}_{2} \times \partial_{\tau}^{2} \mathbf{d}_{2}\right)$

and the constitutive equations read

$v_{3}=\varepsilon_{b} \bar{N}_{3}$
$\overline{\mathbf{M}}=\varepsilon^{2}\left(\bar{\kappa}_{1} \mathbf{d}_{1}+\bar{\kappa}_{2} \mathbf{d}_{2}\right)+\varepsilon^{2} \gamma \bar{\kappa}_{3} \mathbf{d}_{3}$

Dimensionless variables offer the opportunity to compare efficiently the parameters of the problem. A cable is a structural element between a rod and a string. A string is supposed to be perfectly flexible and extensible $(\varepsilon=0)$, contrarily to a rod, a more complex element which is flexible, shearable and extensible. A cable is supposed to be an extensible rod with a small bending stiffness, i.e. $\varepsilon$ is a small parameter. For classical cable structures made up of steel cables, the Young modulus of the material is so high that the ratio $\varepsilon_{b}$ between the axial stiffness and the weight of the cable is also a small parameter.

For the sake of simplicity, the bar over all the dimensionless variables is deliberately omitted in the following developments.

\section{ASYMPTOTIC APPROACH}

\subsection{Outer solution}

The equation of motion in the outer domain is obtained by supposing that the bending effects are small in this region. In this domain, a regular asymptotic expansion in terms of $\varepsilon$ for all variables of the problem reads

$\mathbf{w}=\mathbf{w}_{c}+\varepsilon \mathbf{w}_{c, 1}+\varepsilon^{2} \mathbf{w}_{c, 2}+\ldots$

with $\mathbf{w}(\xi, \tau)$ a generic vector representing the vectors $\mathbf{N}, \mathbf{F}, \mathbf{r}$ or the Euler angles.

After injecting Eq.(15) into Eq.(11) and Eq.(12), the leading order equation reads

$\partial_{\xi}\left(\mathbf{N}_{c}\right)+\mathbf{F}_{c}=\partial_{\tau}^{2} \mathbf{r}_{c}$

with the subscript $c$ referring to the leading order in the outer domain. Equation (16) is the classical form of the equation of motion of extensible strings. The unshearability assumption in the angular momentum equation leads to $N_{c, 1}=N_{c, 2}=0$. Euler angles in the outer domain are $\theta_{c}(\xi, \tau)$ and $\psi_{c}(\xi, \tau)$. The methods to solve this equation are numerous, as finite element method (Impollonia et al. 2011) or modal analysis (Warnitchai et al. 1995). The modal approach is probably less accurate, because it usually neglects the axial displacements and is limited to nonlinear vibrations of taut cables. The modal analysis assumes also synchronous vibration of material points.

\subsection{Inner solution}

The assumption of clamped-clamped beams (Fig.1) explains the existence of two boundary layers at both anchors. To analyse the behaviour of the cable in these two boundary layers, a stretched coordinate $\zeta$ is introduced $\xi=\varepsilon \zeta$, modelling the fast variation of the curvature close to the anchors. This stretched variable $\zeta \in\left[0, \varepsilon^{-1}\right]$ is introduced in the equations of motion. 
For the equilibrium equation in rotation, the rotational inertial forces are three orders of magnitude less than the variation of the bending moment in the boundary layer and they are thus neglected. Equation (12) becomes

$\varepsilon^{-1} \partial_{\zeta} \mathbf{M}+\left(1+v_{3}\right) \mathbf{d}_{3} \times \mathbf{N}=\mathbf{0}$

because of the kinematic equation. The stretched variable $\zeta$ is also introduced in the constitutive equation of $\mathbf{M}$ through the curvature vector $\kappa$, Eq.(10) becomes

$$
\varepsilon \boldsymbol{\kappa}=\left(\cos \theta \partial_{\zeta} \psi+\partial_{\zeta} \phi\right) \mathbf{d}_{3}+\partial_{\zeta} \theta \mathbf{d}_{2}-\sin \theta \partial_{\zeta} \psi \mathbf{d}_{1} .
$$

With Eq.(18) and Eq.(14), Eq.(17) becomes

$$
\begin{cases}\partial_{\zeta}\left(\sin \theta \partial_{\zeta} \psi\right)+\left(1+v_{3}\right) N_{2} & =0 \\ \partial_{\zeta}^{2} \theta+\left(1+v_{3}\right) N_{1} & =0\end{cases}
$$

and $\partial_{\zeta} \kappa_{3}=0$, this latter one meaning that the twist moment is constant along the cable in the boundary layer, $\kappa_{3}=\kappa_{3}^{\star}$. Actually, the twist moment is constant all along the rod, because of the hypothesis of unshearability.

Introducing the stretched variable in Eq.(11) yields

$$
\partial_{\zeta} \mathbf{N}=\varepsilon\left(\partial_{\tau}^{2} \mathbf{r}-\mathbf{F}\right) \text {. }
$$

The right-hand side must be analysed more precisely to evaluate the real order of the inertial forces and the external forces. If no concentrated load acts in the boundary layer and if the external forces are at most of the same order of magnitude as $w l$, the term $\varepsilon \overline{\mathbf{F}}$ is at least of order $\varepsilon$.

The magnitude of the inertial forces requires more attention. The use of the stretched variable leads actually to consider two different problems in the outer and in the inner domains. The matched asymptotic approach is just a method to compose a solution. Therefore, the equation of motion can be considered separately in the boundary layer and in the span. The characteristic time scale in the boundary layer is not the same as in the span. Indeed, the boundary layer may be considered as a cantilever beam with a dimensionless length of $\varepsilon$ (Denoël \& Canor 2013), a unit dead load per unit length and a bending stiffness of $\varepsilon^{2}$. The ratio of the characteristic time in the inner domain $\tau_{b}$ and the one in the outer domain is thus of order $\varepsilon$ (Clough \& Penzien 1993). Because of the smallness of $\varepsilon$, the period $\tau_{b}$ corresponds to the period of a very rigid structure. Using a multiple scales approach in time, the time $\tau$ can be split into two contributions $\tau_{1}=\tau$ related to the span and $\tau_{2}$ related to the boundary layer with $\tau_{2}=\varepsilon \tau$. The inertial forces read now

$\partial_{\tau}^{2} \mathbf{r}=\partial_{\tau_{1}}^{2} \mathbf{r}+2 \varepsilon \partial_{\tau_{1} \tau_{2}}^{2} \mathbf{r}+\varepsilon^{2} \partial_{\tau_{2}}^{2} \mathbf{r}$

and the conservation of linear momentum becomes

$\partial_{\zeta} \mathbf{N}=\varepsilon \partial_{\tau_{1}}^{2} \mathbf{r}+2 \varepsilon^{2} \partial_{\tau_{1} \tau_{2}}^{2} \mathbf{r}-\varepsilon \mathbf{F}+\mathcal{O}\left(\varepsilon^{3}\right)$. with $\mathbf{N}$ now a function of $\tau_{1}, \tau_{2}$ and $\zeta$.

A regular asymptotic expansions in term of $\varepsilon$ for all variables of the problem reads

$\mathbf{u}=\mathbf{u}_{0}+\varepsilon \mathbf{u}_{1}+\varepsilon^{2} \mathbf{u}_{2}+\ldots$

with $\mathbf{u}(\zeta, \tau)$ a generic vector representing the vectors $\mathbf{N}, \mathbf{F}, \mathbf{r}$ or the Euler angles. With Eq.(23) into Eq.(20), the leading order of $\mathbf{N}$ is given by

$\partial_{\zeta} \mathbf{N}_{0}=\mathbf{0}$

which is equivalent to disregard the external forces and the inertial forces in the boundary layer at a lower order of magnitude. This result must be understood with regard to the presumed small dimension of the boundary layer and that the forces are distributed along the cable in a quite regular manner, as the dead load and the inertial forces. The smallness of $\tau_{b}$ compared with $\tau_{1}$ means also a quasi-static response of the boundary layer to an excitation at period close to $\tau_{1}$. This short comment aims to explain intuitively that the boundary layer responds in a quasi-static manner with regard to the fast dynamics of the outer domain.

The leading order of the internal forces $\mathbf{N}_{0}$ can be projected in the leading order basis $\left\{\mathbf{d}_{1,0}, \mathbf{d}_{2,0}, \mathbf{d}_{3,0}\right\}$, and especially

$N_{2}=\mathbf{N}_{0} \cdot \mathbf{d}_{2,0}=-\sin \psi_{0} N_{x}+\cos \psi_{0} N_{y}$

with $N_{x}, N_{y}$ and $N_{z}$ the projections of $\mathbf{N}_{0}$ in a fixed basis $\left\{\mathbf{e}_{x}, \mathbf{e}_{y}, \mathbf{e}_{z}\right\}$. These components are constant in this basis because of Eq.(24). Therefore, $\partial_{\zeta} N_{2}=0$ is only satisfied if $\psi_{0}$ is also constant in the boundary layer. The curvature vector is $\varepsilon \boldsymbol{\kappa}=\partial_{\zeta} \theta_{0} \mathbf{d}_{2,0}+$ $\partial_{\zeta} \phi_{0} \mathbf{d}_{3,0}$ and the angular momentum equation reads

$\partial_{\zeta}^{2} \theta_{0}+\left(1+v_{3}\right) N_{1}=0$

and $N_{2}=0$. So, the constant $\psi_{0} \in[0,2 \pi[$ is determined as $N_{y}=N_{x} \tan \psi_{0}$. The use of reciprocal function leads to discontinuity in the calculation of $\psi_{0}$. From a computational viewpoint, it is more convenient to calculate $\psi_{0}=\arctan \left(\left|\frac{N_{y}}{N_{x}}\right|\right)$ and to adapt $\psi_{0}$ according to the signs of $N_{x}$ and $N_{y}$.

Therefore, the equilibrium equation for the bending moment and the extensibility constitutive equation become

$\partial_{\zeta}^{2} \theta_{0}+\left(1+v_{3}\right)\left(V \cos \theta_{0}-H \sin \theta_{0}\right)=0$

$v_{3}=\varepsilon_{b}\left(V \sin \theta_{0}+H \cos \theta_{0}\right)$

with $V=\left(\cos \psi_{0} N_{x}+\sin \psi_{0} N_{y}\right)$ and $H=N_{z}$. These developments highlight two interesting results which make our approach very elegant : (i) the quasi-static response of the boundary layer avoid the computation of spatial beam dynamics, (ii) the low frequency oscillations in the boundary layer are contained in a plane characterized by the angle $\psi_{0}$, (iii) the torsion is decoupled from the bending dynamics. 
By multiplying Eq.(27) by $\partial_{\zeta} \theta_{0}$, a first integral is found, such as

$$
\begin{aligned}
A(\tau) & =\frac{1}{2}\left(\partial_{\zeta} \theta_{0}\right)^{2}+\left(H \cos \theta_{0}+V \sin \theta_{0}\right) \\
& +\frac{\varepsilon_{b}}{2}\left(\frac{\left(H^{2}-V^{2}\right)}{2} \cos 2 \theta_{0}+V H \sin 2 \theta_{0}\right)
\end{aligned}
$$

with $A(\tau)$ a first integration constant, which is determined by using the van Dyke's rule (Hinch 1991). The matching condition is usually formulated as the inner limit of a given variable equals the outer limit of this variable, such that

$$
\left(\lim _{\varepsilon \rightarrow 0} y_{\text {in }}(\tau, \zeta)\right)_{\xi \text { fixed }}=\left(\lim _{\xi \rightarrow 0} y_{\text {out }}(\tau, \xi)\right)_{\varepsilon \text { fixed }}
$$

with $y$ a variable of the system. This equality leads to identify the internal forces $\mathbf{N}_{0}$ in the boundary layer to the reactions at the anchors calculated with the string dynamics and the angle $\psi_{0}$ to the rotation angle of the string at the anchor, such as

$$
\begin{aligned}
& \lim _{\varepsilon \rightarrow 0, \xi \text { fixed }} \mathbf{N}_{0}(\zeta, \tau)=\lim _{\xi \rightarrow 0, \varepsilon \text { fixed }} \mathbf{N}_{c}(\xi, \tau)=\mathbf{N}_{c, p}(\tau) \\
& \lim _{\varepsilon \rightarrow 0, \xi \text { fixed }} \psi_{0}(\zeta, \tau)=\lim _{\xi \rightarrow 0, \varepsilon \text { fixed }} \Psi(\xi, \tau)=\Psi_{p}(\tau)
\end{aligned}
$$

with the subscript $p$ denoting the anchor at $\xi=0$. Concerning the rotation angle $\theta_{0}$ in the plane orthogonal to $\mathbf{d}_{2}$, we have

$$
\lim _{\varepsilon \rightarrow 0, \xi \text { fixed }} \theta_{0}(\zeta, \tau)=\lim _{\xi \rightarrow 0, \varepsilon \text { fixed }} \vartheta(\xi, \tau)=\vartheta_{p}(\tau)
$$

$$
\lim _{\varepsilon \rightarrow 0, \xi \text { fixed }} \partial_{\zeta} \theta_{0}(\zeta, \tau)=\lim _{\xi \rightarrow 0, \varepsilon \text { fixed }} \partial_{\zeta} \vartheta(\xi, \tau)=0 .
$$

The constant $A(\tau)$ is calculated with these two matching conditions. Furthermore, if we note that $V=$ $H \tan \vartheta_{p}$ which comes from Euler angles in the string dynamics, the equation in $\theta_{0}$ becomes

$$
\frac{1}{2}\left(\partial_{\zeta} \theta_{0}\right)^{2}=2 f_{p} \sin ^{2}\left(\frac{\Delta \theta_{0}}{2}\right)+\varepsilon_{b} \frac{f_{p}^{2}}{2} \sin ^{2}\left(\Delta \theta_{0}\right)
$$

or, in a differential formalism,

$$
\frac{d \theta_{0}}{\sin \left(\frac{\Delta \theta_{0}}{2}\right) \sqrt{1+\varepsilon_{b} f_{p} \cos ^{2}\left(\frac{\Delta \theta_{0}}{2}\right)}}= \pm 2 \sqrt{f_{p}} d \zeta
$$

with $\Delta \theta_{0}=\theta_{0}-\vartheta_{p}$ and $f_{p}(\tau)=\frac{H}{\cos \vartheta_{p}}$. A direct integration leads to

$$
\begin{gathered}
-\frac{1}{\sqrt{1+\varepsilon_{b} f_{p}}} \operatorname{arctanh}\left(\frac{\sqrt{2\left(1+\varepsilon_{b} f_{p}\right)} \cos \left(\frac{\Delta \theta_{0}}{2}\right)}{\sqrt{2+\varepsilon_{b} f_{p}+\varepsilon_{b} f_{p} \cos \left(\Delta \theta_{0}\right)}}\right) \\
= \pm \sqrt{f_{p}} \zeta+B(\tau)
\end{gathered}
$$

with $B(\tau)$ a second integration constant. For usual cable, the term $\varepsilon_{b} f_{p}$ which expresses the ratio between the tension in the cable and the axial stiffness, is small. According to some classical hyperbolic function properties, an explicit expression for $\theta_{0}$ can be approximated by

$$
\begin{aligned}
& \theta_{0}(\zeta, \tau)=\vartheta_{p}(\tau) \\
& \quad+4 \arctan \left(B(\tau) \exp \left( \pm \sqrt{f_{p}\left(1+\varepsilon_{b} f_{p}\right)}\right)\right)
\end{aligned}
$$

Only the minus sign is kept for stability reasons. The constant $B(\tau)$ can be calculated by imposing the rotation angle at the anchor $p$, such that

$B(\tau)=\tan \left(\frac{\theta_{p}-\vartheta_{p}(\tau)}{4}\right)$

with $\theta_{p}=\theta_{0}(0, \tau)$ the imposed boundary condition.

A composed solution can be expressed all along the beam in term of the initial space variable $\xi$, such that

$$
\begin{aligned}
& \theta_{0}(\xi, \tau)=\vartheta(\xi, \tau) \\
& \quad+4 \arctan \left(B(\tau) \exp \left(-\frac{\xi}{\varepsilon} \sqrt{f_{p}\left(1+\varepsilon_{b} f_{p}\right)}\right)\right) \\
& \quad+4 \arctan \left(B(\tau) \exp \left(-\frac{1-\xi}{\varepsilon} \sqrt{f_{q}\left(1+\varepsilon_{b} f_{q}\right)}\right)\right)
\end{aligned}
$$

with the subscript $q$ denoting the anchor at $\xi=1$.

\section{APPLICATIONS}

\subsection{In-plane vibration}

The first application aims at validating the previous developments by studying the response of a horizontal cable submitted to harmonic horizontal displacement of the anchor $q$ (see Figure 1). In this example, the vibrations are contained in the vertical plane to simplify the comparison at this first stage.

The dimensionless properties of the system are $\varepsilon=$ 0.02 and $\varepsilon_{b}=5.6 \cdot 10^{-5}$. The static deflection is characterized by a sag-to-span ratio equal to $5 \%$. The excitation is the function $2 \cdot 10^{-4} \sin \left(2 \omega_{1} \tau\right)$ with $\omega_{1}$ the dimensionless natural circular frequency of the cable equal to 3.3. In this example, a classic analysis with beam finite elements is compared to the proposed approach in which the string dynamic is computed with truss finite elements. The bending moment at the anchors is calculated according to Eq.(40) and $M(\xi)=$ $\varepsilon^{2} \partial_{\xi} \theta_{0}$. In this application, 70 elements are considered for the beam model against 30 for the truss model. Nonlinear corrotational finite elements are used in the numerical simulations. For the beam model, the mesh is adapted to be denser in the boundary regions. Because the number of nodes and the number of DOF per node are reduced, the computational time is drastically reduced between the truss and the beam models. 

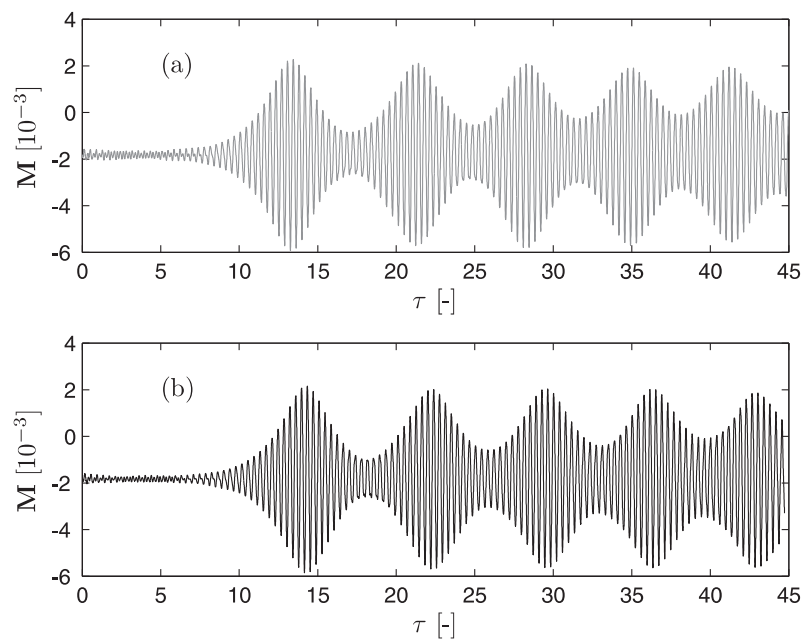

Figure 2: Bending moment $\mathbf{M}\left[10^{-3}\right]$ at the fixed anchor $p$ calculated with the proposed method (a) and a beam finite element model (b).

Furthermore, the instabilities susceptible to appear in the boundary layers are avoided.

Figure 2 highlights the good correspondence between the two methods. The values of the bending moment are identical in both applications, in spite of a short shift between the two time series, explained by the important sensitivity of the transient phase to the natural frequency and the damping model.

\subsection{Spatial vibration}

The second application illustrates the proposed developments for spatial vibrations. The characteristics of the cable are $\varepsilon=0.01, \varepsilon_{b}=10^{-4}$, the sag-to-span ratio is about $5 \%$. The cable is excited by an elliptic displacement of the anchor $q$, such as $x_{q}(\tau)=$ $e_{0, x} \sin \left(\omega_{2} \tau\right)$ and $y_{q}(\tau)=e_{0, y} \sin \left(\omega_{2} \tau\right)$, with $e_{0, x}=$ $10^{-3}$ and $e_{0, y}=e_{0, x} / 2$, with $\omega_{2}$ the first in-plane natural frequency. The outer solution is calculated with only 20 truss finite elements.

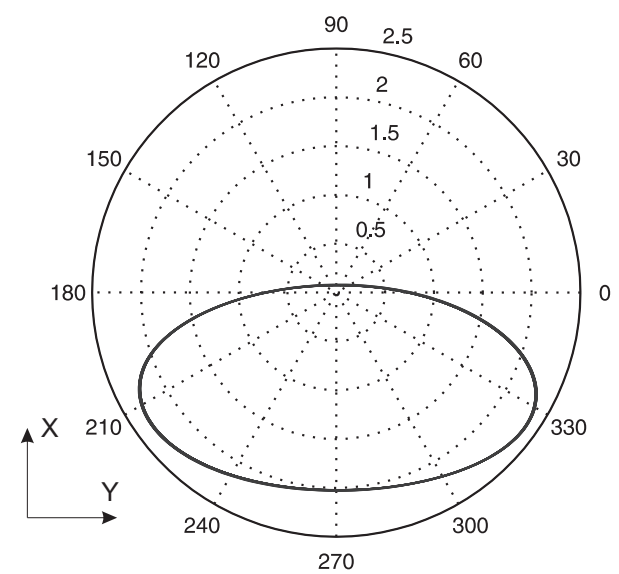

Figure 3: Polar plot of bending moment $\mathbf{M}\left[10^{-3}\right]$ in steady-state vibration at the fixed anchor $p$ for different orientations $\psi_{0}$.

In spatial steady-state vibration, the diagram of the bending moment is adequately shown in a polar graph with the value of the bending moment in the orientation $\psi_{0}(\tau)$ as in Figure 3. For spatial vibrations, the proposed method offers a real efficiency, because

the mesh in the boundary layer must not be refined, the instability due to the twist and the smallness of the bending stiffness are discarded and the number of DOF is reduced (substantial in larger models).

\section{CONCLUSION}

In this paper, an analytical approach using matched asymptotic expansion has been developed. This method allows to calculate the bending moment in the boundary layers by decoupling the equation of motion between the span and the boundary layer. Because these regions respond in a quasi-static way, the proposed formula allows to build the curvature and the bending moment in the boundary layers only by the knowledge of the span vibration.

\section{Acknowledgement}

The authors acknowledge the National Fund of Scientific Research of Belgium for its support.

\section{REFERENCES}

